\title{
Comparison of the Growth and Production of Carps in Polyculture Ponds with Supplemental Feed using Rice Straw and Kanchi as Substrates
}

\author{
S. Rai ${ }^{*}$, Y. Yi ${ }^{1,2}$, Md.A. Wahab ${ }^{3}$, A.N. Bart ${ }^{1}$ and J.S. Diana ${ }^{4}$ \\ ${ }^{1}$ Aquaculture Department, Institute of Agriculture and Animal Science, Rampur, Chitwan, \\ Nepal \\ ${ }^{2}$ College of Aqua-Life Science and Technology, Shanghai Fisheries University, Shanghai, \\ China \\ ${ }^{3}$ Faculty of Fisheries, Bangladesh Agricultural University, Mymensingh, Bangladesh \\ ${ }^{4}$ School of Natural Resources and Environment, University of Michigan, Ann Arbor, \\ Michigan, USA \\ "E-mail:sunilarai@yahoo.com
}

Received: 11.08.2010, Accepted: 06.11.2010

\begin{abstract}
An experiment was carried to compare the performance of rice straw and kanchi in carp polyculture ponds with supplemental feed. The experiment included two treatments in triplicates: a) rice straw substrate $\left(3 \times 625 \mathrm{~kg} \bullet \mathrm{ha}^{-1}\right)$ with supplemental feeding and b) kanchi substrate (390 kanchiøpond $^{-1}$ ) with supplemental feeding. Fingerlings $(\mathrm{n}=40)$ of rohu, Labeo rohita $(23.3 \pm 0.5 \mathrm{~g})$, catla, Catla catla (26.0 $\pm 0.6 \mathrm{~g})$, mrigal, Cirrhinus mrigala $(25.4 \pm 0.7 \mathrm{~g})$, common carp, Cyprinus carpio $(28.5 \pm 1.9 \mathrm{~g})$ and silver carp, Hypophthalmychthys molitrix $(32.1 \pm 1.3 \mathrm{~g})$ were stocked at 3:2:2:2:1 ratio. Fish growth and weight gains did not vary between the rice straw and the kanchi treatment except in catla $(\mathrm{P}>0.05)$. Daily and total weight gains of catla was 48 and $32 \%$ higher in the kanchi treatment than in the rice straw treatment $(\mathrm{P}<0.05)$. However, the rice straw treatment gave more profit than the kanchi treatment. Based on fish production and gross margin, the rice straw treatment seems better for resource-poor farmers.
\end{abstract}

Key words: Rice straw, Kanchi, substrate, supplemental feed, carp polyculture

\section{Introduction}

Substrates added to the ponds indeed Wahab et al., 1999; Keshavnath et al., 2001; increased fish production substantially Azim et al., 2002a; Mridula et al., 2003). It (Hem and Avit, 1994; Ramesh et al., 1999;

is estimated that potential fish production 
from periphyton-based pond aquaculture systems is around 5 tonnes $\bullet$ ha $^{-1} \bullet \mathrm{y}^{-1}$ (Azim et al., 2001b; Van Dam et al., 2002). However, in most cases fish production obtained from periphyton-based aquaculture system is less than the predicted production, and only a few trials (Azim et al., 2001a; Azim et al., 2002a; 2002b) could achieve this fish production level. Van Dam et al. (2002) have suggested that there are three ways to increase fish production in the periphyton-based aquaculture systems: manipulating nutrient levels, using substrates that facilitate periphyton growth, and increasing the surface area index. Since increasing density of bamboo poles beyond a certain level does not increase fish production (Keshavnath et al., 2002; Azim et al., 2004), it is not economically feasible to increase substrate density further. Thus, manipulating nutrient levels and using substrates that facilitate periphyton growth seem to be possible solution to enhance fish production in periphyton-based pond aquaqculture system.

In semi-intensive systems, artificial feed benefits the ponds in two ways either through direct consumption by cultured fish or indirect supply of nutrients from decomposition by benthos, fungi and protozoa (Moriarty, 1986; Milstein, 1992; Moriarty, 1997). In pond culture, on average about $21 \%$ of nitrogen and $19 \%$ of phosphorous in the artificial feed are retained by the fish (Siddiqui and Al-Harbi, 1999), while $14 \%$ of nitrogen and $21 \%$ of phosphorous are used by phytoplankton (Neori and Krom, 1991) and the remaining nitrogen and phosphorous mainly stimulate bacteria, fungi and protozoa production, which in turn may be consumed by zooplankton (Tang, 1970; Langis et al., 1988). Since bamboo is expensive to resource-poor farmers, rice straw and kanchi can be alternatives to bamboo. Previous studies on rice straw (Ramesh et al., 1999; Mridula et al., 2003; Mridula et al., 2005) and kanchi (Wahab et al., 1999; Azim et al., 2002a) as substrates in non-fed ponds have showed that both substrates are capable to enhance fish production. There is a need to compare the performance of rice straw and kanchi in carp polyculture ponds with supplemental feed.

The objective of the experiment was to compare the effect of rice straw and kanchi on water quality, plankton, periphyton, benthos, bacteria, carp growth and production, and economic returns in carp polyculture ponds supplemented with on-farm feed.

\section{Materials and methods}

An experiment was conducted in six $40 \mathrm{~m}^{-2}$ $(8 \times 5 \mathrm{~m})$ ponds of $1.5 \mathrm{~m}$ deep at Field Laboratory of Fisheries Faculty, Bangladesh Agricultural University at Mymensingh, Bangladesh for 90 days during February to May 2006. The experiment included two treatments in triplicates each: a) rice straw substrate ( 3 straw mats per pond, $3 \times 625 \mathrm{~kg} \bullet$ $\mathrm{ha}^{-1}$ ) with supplemental feed (rice straw treatment) and b) kanchi substrate (390 kanchi per pond) with supplemental feed (kanchi treatment). The treatments were allocated to the experimental ponds randomly.

Prior to placing the substrates, all ponds were drained and dried for 10 days. The ponds were limed with $\mathrm{CaO}$ at a rate of $250 \mathrm{~kg} \bullet \mathrm{ha}^{-1}$. Three days later the ponds were filled to $0.30 \mathrm{~m}$ deep. Afterwards, 390 kanchi $(1.5 \mathrm{~cm}$ in diameter and $1.8 \mathrm{~m}$ in length) and three rice straw mats $(2 \times 1 \mathrm{~m})$ were fixed in each of the kanchi and rice straw treatment ponds, respectively. Rice 
straw mats were prepared by pressing rice straw bundles between bamboo splits. Then, all ponds were filled to $1.10 \mathrm{~m}$ deep. Following day, ponds were fertilized with urea, triple superphosphate (TSP) and cow dung at rates of $31 \mathrm{~kg} \bullet \mathrm{ha}^{-1}, 16 \mathrm{~kg} \bullet \mathrm{ha}^{-1}$ and $1,250 \mathrm{~kg} \bullet \mathrm{ha}^{-1}$, respectively, at fortnight basis. Dissolved oxygen (DO) in the rice straw ponds was monitored for two weeks until it reached the level higher than 2.2 $\mathrm{mg} \cdot \mathrm{L}^{-1}$ in the ponds. Then, fingerlings of rohu $(23.3 \pm 0.5 \mathrm{~g})$, mrigal $(25.4 \pm 0.7 \mathrm{~g})$, catla $(26.0 \pm 0.6 \mathrm{~g})$, silver carp $(32.1 \pm 1.3 \mathrm{~g})$ and common carp $(28.5 \pm 1.9 \mathrm{~g})$ were stocked at a species ratio of 3:2:2:2:1 and a density of 1 fish $\bullet \mathrm{m}^{-2}$. Supplementary feed made of rice bran and mustard oil cake (60:40) was given to fish. The feeding rate was kept at $3 \%$ of the body weight.

Dissolved oxygen, temperature and $\mathrm{pH}$ were measured weekly at 0600, 1800 and $0600 \mathrm{~h}$ of next day using a YSI model 58 oxygen meter (Yellow Springs Instruments, Yellow Springs, OH, USA) and a $\mathrm{pH}$ meter (HANA Microelectronics Public Co. Ltd., Bangkok, Thailand). DO concentrations were measured at three depths, $10 \mathrm{~cm}, 50 \mathrm{~cm}$ and $70 \mathrm{~cm}$ below water surface. Secchi disc depth was monitored weekly at $0900 \mathrm{~h}$. Composite column water samples were collected monthly at $0900-1000 \mathrm{~h}$ from three locations of each pond to analyse total alkalinity, total ammonia nitrogen (TAN), nitrite-nitrogen, soluble reactive phosphorus (SRP), total phosphorus (TP), chlorophyll- $a$, total suspended solids (TSS) and total volatile solids (TVS) following APHA (1980) and total nitrogen (TN) following Raveh and Avnimelech (1979).

Composite column water samples were also collected monthly for the analyses of planktons. A 5-L of sampled water was passed through plankton net with mesh size of $25 \mu \mathrm{m}$ to make a concentrated volume of $50 \mathrm{~mL}$. The concentrated samples were preserved in small plastic bottles containing $6 \%$ formalin. Planktons were enumerated using a Sedgewick-Rafter counting cell (S$\mathrm{R}$ cell) under a binocular microscope (Swift M-4000, Swift Instrument Inc.). Plankton concentrations were estimated using the following formula: $\mathrm{N}=(\mathrm{P} \times \mathrm{C} \times 100) / \mathrm{L}$. where, $\mathrm{N}=$ the number of plankton units per litre of original pond water; $\mathrm{P}=$ the number of planktons counted in ten random fields of $\mathrm{S}-\mathrm{R}$ cell; $\mathrm{C}=$ the volume of final concentrated sample $(\mathrm{mL}) ; \mathrm{L}=$ the volume (L) of the pond water sample.

Pieces of rice straw was cut by scissors from three different depths (surface, middle and bottom) of each mat from each replication, and wrapped in an aluminum foil for monthly periphyton analysis. Each sample was transferred to an Erlenmeyer flask containing $50-\mathrm{mL}$ distilled water and shaken in a mechanical shaker for 3 hours to detach periphytons from the straw surface. After removing periphytons from straw, the straw was dried overnight in an oven at $80^{\circ} \mathrm{C}$ to get the dry weight. For taxonomic identification, samples were preserved in $6 \%$ formalin. Periphytons were counted using a S-R cell under a binocular microscope. The number of periphyton units was estimated by using following formula: $\mathrm{N}=(\mathrm{P} \times \mathrm{C} \times 100) / \mathrm{W}$. where, $\mathrm{N}=$ Number of periphyton units; $\mathrm{P}=$ Number of periphyton units counted in ten random fields of S-R cell; $\mathrm{C}=$ Volume of final concentrated sample $(\mathrm{mL}) ; \mathrm{W}=$ Weight of rice straw $(\mathrm{g})$

Periphyton taxa were identified to genus level by using keys from Ward and Whipple (1959), Wetzel (1983) and Bellinger (1992). 
Dry matter of periphytons was estimated by filtering samples through preweighed and oven-dried GF/C filter papers and drying for 24 hours in an oven at $105^{\circ} \mathrm{C}$. It was further combusted in a Muffle furnace at $550^{\circ} \mathrm{C}$ for $30 \mathrm{~min}$ to get ash content (\%). Chlorophyll- $a$ concentration was determined following the standard methods (APHA 1980). Periphytons from kanchi were analysed following Azim et al. (2002a).

Pieces of rice straw was cut from three different depths of each mat, pooled and kept in a sterilized tube containing phosphate buffer solution for bacteria analysis. Samples from the kanchi were collected by scrapping $2 \times 2 \mathrm{~cm}^{2}$ area by scalpel, and kept in a sterilized tube containing phosphate buffer solution. The samples were preserved in a refrigerator at $4^{\circ} \mathrm{C}$. Total plate counting of the bacteria was done following APHA (1980). Periphyton number, biomass and bacteria total plate count were estimated based on the pond area for comparison between treatments.

Zoobenthic samples from the bottom of each pond were collected monthly by using an Ekman dredge $(15 \times 15 \mathrm{~cm})$. The mud samples were collected from three random locations. The content of the dredge was sieved through a sieve of $250 \mu \mathrm{m}$ mesh size. Zoobenthos were separated and preserved in $10 \%$ formalin. Zoobenthos were identified under a dissecting microscope (CH40RF200 Model, Olympus, Japan) following keys from Ward and Whipple (1959) and Needham and Needham (1962). Zoobenthos number was estimated following Rahman et al. (2006). $\mathrm{N}=(\mathrm{Y} \times 10,000) / 3 \mathrm{~A}$. Where, $\mathrm{N}=$ number of benthic organisms per square meter (individuals $\bullet \mathrm{m}^{-2}$ ); $\mathrm{Y}=$ total number of benthic organisms counted in 3 samples; $\mathrm{A}=$ area of Ekman dredge $\left(\mathrm{cm}^{2}\right)$.

At least $30 \%$ of each stocked fish species were sampled monthly and weighed individually using an electronic scale to determine fish growth and adjust amount of feed. At the end, substrates were removed from the ponds, and fish were harvested, counted and weighed individually. Weight gains and survival rates were calculated.

Gross margin analysis was carried out to compare economic returns between treatments. The prices of all inputs and outputs were based on the local market price at Mymensingh. The analysis excluded labour cost as rural farmers use family labours to get farm work done. Expectant life of bamboo and kanchi was assumed to be 3 , and 1 and half years, respectively.

Data were statistically analyzed by Student's t-test using SPSS (version 12.0) statistical software (SPSS Inc., Chicago, USA). Differences were considered significant at an alpha level of 0.05 $(p<0.05)$. All means were given with \pm 1 standard error (S.E.).

\section{Result}

There were no significant differences in the water quality parameters between treatments except DO concentration (P>0.05, Tab. 1). DO concentration at 0600 $\mathrm{h}$ was significantly lower at three depths in the rice straw treatment than that in the kanchi treatment $(\mathrm{P}<0.05)$, while DO concentration at $1800 \mathrm{~h}$ was significantly lower only at $10 \mathrm{~cm}$ below water surface in the rice straw treatment than that in the kanchi treatment $(\mathrm{P}<0.05)$.

Phytoplankton and zooplankton densities in pond water did not differ between treatments ( $P>0.05$, Tab. 2-3). Density of zoobenthos in the pond sediment 
was $512 \pm 165$ individual $\bullet \mathrm{m}^{-2}$ in the rice straw and $280 \pm 34$ individual $\bullet \mathrm{m}^{-2}$ in the kanchi treatments, which were not significantly different $(\mathrm{P}>0.05)$.

Periphyton densities didn't differ significantly between treatments $(\mathrm{P}>0.05$, Tab. 4). Dry matter, ash, ash free dry matter and chlorophyll-a concentration of periphytons did not differ significantly between treatments $(\mathrm{P}>0.05$, Tab. 5). Bacteria total plate count was $65,460 \pm 11,620\left(\times 10^{6} \mathrm{cfu} \cdot \mathrm{m}^{-2}\right)$ in the rice straw, which was higher than that of $13,035 \pm 1,202\left(\times 10^{6} \mathrm{cfu} \cdot \mathrm{m}^{-2}\right)$ in the kanchi $(\mathrm{P}<0.05)$.

There were no significant differences in fish growth and production except catla between the rice straw and kanchi treatments $(\mathrm{P}>0.05$, Tab. 6). Daily and total weight gains of catla were higher in the kanchi treatment than in the rice straw treatment $(\mathrm{P}<0.05)$. Combined total weight gain was also did not differ between the rice straw and kanchi treatment $(\mathrm{P}>0.05)$. Silver carp (24-32\%) and rohu (23-27\%) were the major contributors to combined total weight gain in all three treatments while mrigal, common carp and catla contributed $16-22 \%$, $11-20 \%$ and $12-15 \%$ respectively (Fig. 1). There were no differences in survival rates of rohu, catla, mrigal, common carp and silver carp among all treatments $(\mathrm{P}>0.05)$. Overall FCR didn't differ between the rice straw and kanchi treatments $(\mathrm{P}>0.05)$.

Gross margin analysis showed that gross return was significantly higher in the kanchi treatment than in the rice straw treatment $(\mathrm{P}<0.05 ;$ Tab. 7). In contrast, gross margin was higher in the rice straw treatment than in the kanchi treatment.

\section{Discussion}

All water quality parameters remained in the normal range for carp culture. There were no significant effects of substrates on water quality except on dissolved oxygen. Low dissolved oxygen concentration in the rice straw treatment was probably due to increased biological oxygen demand (Dharmaraj et al., 2002) which is common in the water with predominate heterotrophic food production (Moriarity, 1997).

Adding substrate to the fed ponds did not affect densities of plankton and zoobenthos significantly in the present experiment. Plankton abundance in pond water showed the fertile state of the ponds in all treatments. Abundance of zoobenthos was in agreement with the result reported by Habib et al. (1984) in BAU ponds. Periphyton density and biomass did not differ between rice straw and kanchi, indicating that both substrates were equally preferred by periphytons. Contrast to periphytons, bacteria preferred rice straw over kanchi. Higher bacteria total plate count on the rice straw was perhaps due to the provision of more organic matter and surface area for bacterial growth (Schroeder, 1978; Van Dam et al., 2002).

Fish growth and production did not vary between the rice straw and kanchi treatments except catla, indicating that both substrates favour growth and production of carps. Growth rate was higher than 1.2 $\mathrm{g} \cdot$ fish $^{-1} \cdot$ day $^{-1}$ in all species in both treatments, except in catla in the rice straw treatment. Lower daily and total weight gains of catla in the rice straw treatment than in the kanchi treatment could be attributed to relatively lower zooplankton population in the rice straw ponds with supplemental feed. Catla is predominantly zooplankton feeder (Chakrabarti, 1998). In 
S. Rai, Y.Yi, Md.A. Wahab, A.N. Bart and J.S. Diana / Our Nature (2010) 8: 92-105

Table 1. Summary of water quality parameters in the rice straw and kanchi treatments (Mean \pm S.E.)

\begin{tabular}{|c|c|c|}
\hline Parameter & Rice straw & Kanchi \\
\hline Temperature at $0600 \mathrm{~h}\left({ }^{\circ} \mathrm{C}\right)$ & $25.8 \pm 1.0$ & $26.3 \pm 0.0$ \\
\hline Temperature at $1800 \mathrm{~h}\left({ }^{0} \mathrm{C}\right)$ & $29.4 \pm 0.0$ & $29.4 \pm 0.1$ \\
\hline \multicolumn{3}{|l|}{$\overline{\mathrm{DO}}$ at $0600 \mathrm{~h}\left(\mathrm{mg} \cdot \mathrm{L}^{-1}\right)$} \\
\hline $10 \mathrm{~cm}$ & $3.8 \pm 0.1^{\mathrm{b}}$ & $5.0 \pm 0.1^{\mathrm{a}}$ \\
\hline $50 \mathrm{~cm}$ & $3.2 \pm 0.1^{\mathrm{b}}$ & $4.2 \pm 0.1^{\mathrm{a}}$ \\
\hline $70 \mathrm{~cm}$ & $2.8 \pm 0.1^{\mathrm{b}}$ & $3.7 \pm 0.1^{\mathrm{a}}$ \\
\hline \multicolumn{3}{|l|}{$\mathrm{DO}$ at $1800 \mathrm{~h}\left(\mathrm{mg} \cdot \mathrm{L}^{-1}\right)$} \\
\hline $10 \mathrm{~cm}$ & $7.5 \pm 0.2^{\mathrm{b}}$ & $9.2 \pm 0.1^{\mathrm{a}}$ \\
\hline $50 \mathrm{~cm}$ & $6.62 \pm 1.0$ & $7.5 \pm 0.2$ \\
\hline $70 \mathrm{~cm}$ & $4.7 \pm 0.3$ & $5.6 \pm .0 .3$ \\
\hline $\mathrm{pH}$ at $0600 \mathrm{~h}$ & $8.4 \pm 0.0$ & $8.4 \pm 0.0$ \\
\hline $\mathrm{pH}$ at $1800 \mathrm{~h}$ & $8.8 \pm 0.0$ & $8.8 \pm 0.0$ \\
\hline Secchi disk depth $(\mathrm{cm})$ & $24.3 \pm 0.8$ & $21.53 \pm 0.8$ \\
\hline Total alkalinity $\left(\mathrm{mg} \cdot \mathrm{L}^{-1}\right.$ as $\left.\mathrm{CaCO}_{3}\right)$ & $125 \pm 5$ & $143 \pm 7$ \\
\hline Chlorophyll-a (ug・ $\left.\mathrm{L}^{-1}\right)$ & $50 \pm 11$ & $61 \pm 13$ \\
\hline Total nitrogen $\left(\mathrm{mg} \cdot \mathrm{L}^{-1}\right)$ & $1.45 \pm 0.27$ & $1.42 \pm 0.17$ \\
\hline Total ammonium nitrogen $\left(\mathrm{mg} \cdot \mathrm{L}^{-1}\right)$ & $0.10 \pm 0.01$ & $0.15 \pm 0.03$ \\
\hline Nitrite nitrogen $\left(\mathrm{mg} \cdot \mathrm{L}^{-1}\right)$ & $0.01 \pm 0.00$ & $0.01 \pm 0.01$ \\
\hline Total phosphorous $\left(\mathrm{mg} \cdot \mathrm{L}^{-1}\right)$ & $1.94 \pm 0.05$ & $2.16 \pm 0.37$ \\
\hline Soluble reactive phosphorous $\left(\mathrm{mg} \cdot \mathrm{L}^{-1}\right)$ & $0.90 \pm 0.16$ & $0.87 \pm 0.28$ \\
\hline Total suspended solids $\left(\mathrm{mg} \cdot \mathrm{L}^{-1}\right)$ & $75 \pm 1$ & $74 \pm 9$ \\
\hline Total volatile solids $\left(\mathrm{mg} \cdot \mathrm{L}^{-1}\right)$ & $46 \pm 3$ & $44 \pm 8$ \\
\hline
\end{tabular}

Mean values with different superscript letters in the same row are significantly different $(\mathrm{P}<0.05)$.

Table 2. Abundance of phytoplankton (units $\bullet \mathrm{L}^{-1}$ ) in the pond water in the rice straw and kanchi treatments (Mean \pm SE)

\begin{tabular}{llll}
\hline Group & Genus & Rice straw & Kanchi \\
\hline Bacillariophyceae & Coscinodiscus & $2,302 \pm 831$ & $2,062 \pm 387$ \\
Cyclotella & $10,135 \pm 6,131$ & $55,998 \pm 49,845$ \\
Diatoma & $667 \pm 406$ & $450 \pm 158$ \\
Fragillaria & $3,202 \pm 946$ & $2,255 \pm 1,780$ \\
Gomphonema & $370 \pm 186$ & $903 \pm 423$ \\
Gyrosigma & $0 \pm 0$ & $85 \pm 85$ \\
Melosira & $3,458 \pm 648$ & $6,183 \pm 3,221$ \\
Navicula & $3,043 \pm 1,749$ & $1,608 \pm 394$ \\
Nitzschia & $5,818 \pm 2,068$ & $2,775 \pm 1,003$ \\
Pinnularia & $0 \pm 0$ & $0 \pm 0$ \\
Surirella & $975 \pm 333$ & $860 \pm 206$ \\
Synedra & $4,575 \pm 3,718$ & $823 \pm 192$ \\
Tabellaria & $872 \pm 757$ & $542 \pm 85$ \\
Subtotal & $35,417 \pm 4,600$ & $74,548 \pm 46,077$ \\
\hline Chlorophyceae & Actinastrum & $572 \pm 72$ & $392 \pm 173$ \\
& Centritractus & $322 \pm 161$ & $418 \pm 215$ \\
& & $0 \pm 0$ & $167 \pm 167$ \\
\end{tabular}


S. Rai, Y.Yi, Md.A. Wahab, A.N. Bart and J.S. Diana / Our Nature (2010) 8: 92-105 Table 2-Contd....

\begin{tabular}{|c|c|c|c|}
\hline & \multirow{4}{*}{$\begin{array}{l}\text { Chlamydomonas } \\
\text { Characium } \\
\text { Chlorella }\end{array}$} & \multirow{3}{*}{$\begin{array}{l}412 \pm 217 \\
0 \pm 0\end{array}$} & \multirow[b]{2}{*}{$255 \pm 3$} \\
\hline & & & \\
\hline & & & $0 \pm 0$ \\
\hline & & $8,933 \pm 2,807$ & $14,748 \pm 1,262$ \\
\hline & Chodatella & $42 \pm 42$ & $90 \pm 90$ \\
\hline & Coelastrum & $0 \pm 0$ & $1,433 \pm 1,192$ \\
\hline & Cosmarium & $45 \pm 45$ & $0 \pm 0$ \\
\hline & Crucigenia & $4,267 \pm 1,592$ & $5,152 \pm 2,034$ \\
\hline & Gonatozygon & $95 \pm 95$ & $487 \pm 177$ \\
\hline & Microspora & $177 \pm 177$ & $587 \pm 463$ \\
\hline & Mougeotia & $3,662 \pm 1,690$ & $7,738 \pm 1,665$ \\
\hline & Oedogonium & $0 \pm 0$ & $85 \pm 85$ \\
\hline & Oocystis & $4,850 \pm 488$ & $3,648 \pm 705$ \\
\hline & Pediastrum & $2,617 \pm 1,634$ & $4,063 \pm 1,805$ \\
\hline & Scenedesmus & $8,287 \pm 2,237$ & $11,858 \pm 1,830$ \\
\hline & Selenastrum & $625 \pm 317$ & $152 \pm 77$ \\
\hline & Sphaerocystis & $2,043 \pm 732$ & $9,602 \pm 5,677$ \\
\hline & Staurastrum & $78 \pm 78$ & $237 \pm 237$ \\
\hline & Tetraspora & $737 \pm 186$ & $777 \pm 420$ \\
\hline & Tetraedron & $615 \pm 315$ & $575 \pm 163$ \\
\hline & Treubaria & $157 \pm 157$ & $157 \pm 157$ \\
\hline & Ulothrix & $357 \pm 229$ & $933 \pm 933$ \\
\hline & Volvox & $167 \pm 84$ & $83 \pm 83$ \\
\hline & Subtotal & $45,625 \pm 8,535$ & $57,830 \pm 3,475$ \\
\hline \multirow[t]{9}{*}{ Cyanophyceae } & Anabaena & $168 \pm 85$ & $212 \pm 149$ \\
\hline & Aphanocapsa & $78 \pm 78$ & $0 \pm 0$ \\
\hline & Chroococcus & $1,537 \pm 755$ & $2,595 \pm 460$ \\
\hline & Gloecapsa & $1,513 \pm 573$ & $463 \pm 247$ \\
\hline & Gomphosphaeria & $823 \pm 147$ & $535 \pm 283$ \\
\hline & Merismopedia & $0 \pm 0$ & $160 \pm 160$ \\
\hline & Microcystis & $420 \pm 289$ & $467 \pm 154$ \\
\hline & Oscillatoria & $1,642 \pm 1,066$ & $348 \pm 176$ \\
\hline & Subtotal & $6,182 \pm 1,469$ & $4,780 \pm 697$ \\
\hline \multirow[t]{4}{*}{ Euglenophyceae } & Euglena & $41,170 \pm 13,606$ & $109,355 \pm 70,951$ \\
\hline & Phacus & $1,163 \pm 144$ & $1,122 \pm 286$ \\
\hline & Trachelomonas & $800 \pm 113$ & $1,245 \pm 530$ \\
\hline & Subtotal & $43,133 \pm 13359$ & $111,722 \pm 71,165$ \\
\hline Total phytoplankton & & $130,357 \pm 16,683$ & $248,880 \pm 114,423$ \\
\hline Identified genus (no.) & & 42 & 45 \\
\hline
\end{tabular}


S. Rai, Y.Yi, Md.A. Wahab, A.N. Bart and J.S. Diana / Our Nature (2010) 8: 92-105

Table 3. Abundance of zooplankton (units $\bullet \mathrm{L}^{-1}$ ) in the pond water in the rice straw and kanchi treatments

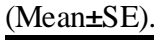

\begin{tabular}{llll}
\hline Group & Genus & Rice straw & Kanchi \\
\hline Sarcodina & Difflugia & $715 \pm 271$ & $915 \pm 369$ \\
\hline Rotifera & Asplanchna & $902 \pm 42$ & $828 \pm 225$ \\
Brachionus & $1,872 \pm 341$ & $2,142 \pm 597$ \\
& Filinia & $140 \pm 71$ & $580 \pm 300$ \\
Keratella & $693 \pm 250$ & $572 \pm 188$ \\
& Lecane & $227 \pm 129$ & $567 \pm 289$ \\
& Monostyla & $0 \pm 0$ & $140 \pm 140$ \\
& Polyarthra & $1,453 \pm 619$ & $1,237 \pm 395$ \\
& Trichocerca & $473 \pm 125$ & $587 \pm 308$ \\
& Subtotal & $5,760 \pm 922$ & $6,652 \pm 1553$ \\
\hline Crustacea & Cyclops & $842 \pm 164$ & $935 \pm 349$ \\
& Diaptomus & $290 \pm 75$ & $152 \pm 152$ \\
& Ceriodaphnia & $222 \pm 117$ & $297 \pm 97$ \\
& Daphnia & $150 \pm 150$ & $142 \pm 71$ \\
& Diaphanosoma & $157 \pm 157$ & $0 \pm 0$ \\
& Moina & $327 \pm 60$ & $525 \pm 351$ \\
& Nauplius & $1,530 \pm 153$ & $1,535 \pm 311$ \\
\hline Tdentified species (no.) & Subtotal & $3,517 \pm 453$ & $3,585 \pm 840$ \\
\hline
\end{tabular}

Table 4. Abundance of periphyton $\left(10^{3} \times\right.$ units $\left.\bullet \mathrm{m}^{-2}\right)$ in the rice straw and kanchi treatments $($ Mean $\pm \mathrm{SE})$

\begin{tabular}{llll}
\hline Group & Genus & Rice straw & Kanchi \\
\hline Bacillariophyceae & Coscinodiscus & $1,068 \pm 1,068$ & $868 \pm 459$ \\
& Cyclotella & $2,426 \pm 445$ & $3,471 \pm 1,138$ \\
Cymbella & $560 \pm 560$ & $0 \pm 0$ \\
Diatoma & $16,221 \pm 8,258$ & $20,476 \pm 10,326$ \\
Fragillaria & $43,367 \pm 9,405^{\mathrm{a}}$ & $14,750 \pm 4,211^{\mathrm{b}}$ \\
& Gomphonema & $9,585 \pm 1,679^{\mathrm{a}}$ & $174 \pm 174^{\mathrm{b}}$ \\
Melosira & $12,258 \pm 984$ & $6,073 \pm 4,002$ \\
& Navicula & $37,309 \pm 8,922$ & $74,096 \pm 23,699$ \\
& Nitzschia & $80,142 \pm 25,592$ & $55,182 \pm 22,538$ \\
& Surirella & $513 \pm 513$ & $0 \pm 0$ \\
Synedra & $33,539 \pm 4,440^{\mathrm{a}}$ & $12,494 \pm 1,673^{\mathrm{b}}$ \\
Tabellaria & $5,492 \pm 2,859$ & $1,735 \pm 459$ \\
Subtotal & $242,482 \pm 30,886$ & $189,319 \pm 21,005$ \\
\hline Chlorophyceae & Actinastrum & $1,120 \pm 560$ & $0 \pm 0$ \\
& Centritractus & $560 \pm 560$ & $174 \pm 174$ \\
& Characium & $1,943 \pm 1,205$ & $5,726 \pm 2,671$ \\
& & & Contd....
\end{tabular}


S. Rai, Y.Yi, Md.A. Wahab, A.N. Bart and J.S. Diana / Our Nature (2010) 8: 92-105 Table 4-Contd....

\begin{tabular}{|c|c|c|c|}
\hline & Chlorella & $46,942 \pm 16,399$ & $7,115 \pm 1,483$ \\
\hline & Closterium & $1,719 \pm 318$ & $694 \pm 459$ \\
\hline & Coelastrum & $0 \pm 0$ & $868 \pm 347$ \\
\hline & Cosmarium & $3,267 \pm 1,587$ & $174 \pm 174$ \\
\hline & Crucigenia & $23,277 \pm 12,917$ & $2,429 \pm 1,655$ \\
\hline & Cylindrocapsa & $0 \pm 0$ & $14,229 \pm 3,623$ \\
\hline & Gonatozygon & $5,090 \pm 2,239$ & $0 \pm 0$ \\
\hline & Microspora & $2,377 \pm 2,377$ & $694 \pm 347$ \\
\hline & Mougeotia & $20,511 \pm 7,717$ & $2,082 \pm 902$ \\
\hline & Oedogonium & $16,139 \pm 9,598$ & $10,759 \pm 5,968$ \\
\hline & Oocystis & $1,531 \pm 149^{\mathrm{b}}$ & $3,471 \pm 626^{\mathrm{a}}$ \\
\hline & Pediastrum & $7,556 \pm 2,828$ & $1,388 \pm 626$ \\
\hline & Scenedesmus & $44,991 \pm 8,687$ & $22,732 \pm 7,064$ \\
\hline & Selenastrum & $560 \pm 560$ & $0 \pm 0$ \\
\hline & Stigeoclonium & $762 \pm 762^{b}$ & $180,469 \pm 46,057^{\mathrm{a}}$ \\
\hline & Staurastrum & $0 \pm 0$ & $174 \pm 174$ \\
\hline & Tetraspora & $0 \pm 0$ & $174 \pm 174$ \\
\hline & Tetraedorn & $1,120 \pm 560$ & $347 \pm 174$ \\
\hline & Triplocerus & $3,658 \pm 2,047$ & $0 \pm 0$ \\
\hline & Ulothrix & $1,480 \pm 933$ & $0 \pm 0$ \\
\hline & Subtotal & $184,603 \pm 40,741$ & $253,697 \pm 40,753$ \\
\hline Cyanophyceae & Chroococcus & $2,194 \pm 2,194$ & $2,429 \pm 1,655$ \\
\hline & Gloecapsa & $0 \pm 0$ & $4,685 \pm 1,562$ \\
\hline & Gomphosphaeria & $411 \pm 411$ & $0 \pm 0$ \\
\hline & Oscillatoria & $10,633 \pm 3,422$ & $10,932 \pm 10,161$ \\
\hline & Phormidium & $12,151 \pm 3,421$ & $2,776 \pm 347$ \\
\hline & Subtotal & $25,389 \pm 3,837$ & $20,823 \pm 9,097$ \\
\hline Euglenophyceae & Euglena & $10,113 \pm 146^{\mathrm{a}}$ & $694 \pm 347^{b}$ \\
\hline & Phacus & $560 \pm 560$ & $0 \pm 0$ \\
\hline & Trachelomonas & $1,485 \pm 938$ & $174 \pm 174$ \\
\hline & Subtotal & $12,158 \pm 1,162^{\mathrm{a}}$ & $868 \pm 459^{b}$ \\
\hline Sarcodina & Difflugia & $3,352 \pm 613$ & $521 \pm 0$ \\
\hline$\overline{\text { Rotifera }}$ & Asplanchna & $1,528 \pm 765$ & $1,041 \pm 601$ \\
\hline & Brachionus & $1,027 \pm 1,027$ & $347 \pm 347$ \\
\hline & Conochilus & $822 \pm 822$ & $1,735 \pm 966$ \\
\hline & Lecane & $762 \pm 762$ & $521 \pm 301$ \\
\hline & Monostyla & $0 \pm 0$ & $347 \pm 174$ \\
\hline & Subtotal & $4,139 \pm 903$ & $3,991 \pm 1,215$ \\
\hline Total Periphyton & & $472,123 \pm 71,506$ & $469,218 \pm 31,646$ \\
\hline Identified genus (no.) & & 43 & 40 \\
\hline
\end{tabular}

Mean values with different superscript letters in the same row are significantly different $(\mathrm{P}<0.05)$. 
S. Rai, Y.Yi, Md.A. Wahab, A.N. Bart and J.S. Diana / Our Nature (2010) 8: 92-105

Table 5. Periphyton biomass and pigment concentration in the rice straw and kanchi treatments (Mean \pm S.E.)

\begin{tabular}{|c|c|c|}
\hline Parameter & Rice straw & Kanchi \\
\hline$\overline{\text { Dry matter }\left(\mathrm{g} \bullet \text { pond }^{-1}\right)}$ & $634.4 \pm 14.4$ & $803.9 \pm 163.7$ \\
\hline Ash (\%) & $43.3 \pm 2.0$ & $45.9 \pm 1.7$ \\
\hline Ash Free Dry Matter $\left(\mathrm{g} \bullet\right.$ pond $\left.^{-1}\right)$ & $302.0 \pm 19.8$ & $432.6 \pm 64.6$ \\
\hline Chlorophyll-a $\left(\mathrm{g} \bullet\right.$ pond $\left.^{-1}\right)$ & $1.1 \pm 0.1$ & $1.9 \pm 0.3$ \\
\hline
\end{tabular}

Table 6. Growth Performance of carps stocked in the rice straw and kanchi treatments (Mean \pm S.E.)

\begin{tabular}{|c|c|c|}
\hline Parameter & Rice straw & Kanchi \\
\hline \multicolumn{3}{|l|}{$\overline{\text { Rohu }}$} \\
\hline Initial total weight $\left(\mathrm{kg} \bullet\right.$ pond $\left.^{-1}\right)$ & $0.28 \pm 0.01$ & $0.28 \pm 0.01$ \\
\hline Initial mean weight $\left(\mathrm{g} \bullet \mathrm{fish}^{-1}\right)$ & $23.10 \pm 0.47$ & $23.53 \pm 1.21$ \\
\hline Final total weight $\left(\mathrm{kg}^{\circ}\right.$ pond $\left.^{-1}\right)$ & $1.73 \pm 0.12$ & $1.89 \pm 0.05$ \\
\hline Final mean weight $\left(\mathrm{g} \bullet \mathrm{fish}^{-1}\right)$ & $150.30 \pm 4.09$ & $162.07 \pm 8.85$ \\
\hline Daily weight gain $\left(\mathrm{g} \bullet \mathrm{fish}^{-1} \bullet\right.$ day $\left.^{-1}\right)$ & $1.41 \pm 0.05$ & $1.54 \pm 0.10$ \\
\hline Total weight gain $\left(\mathrm{kg}^{\circ}\right.$ pond $\left.^{-1}\right)$ & $1.45 \pm 0.12$ & $1.60 \pm 0.06$ \\
\hline Survival (\%) & $100.0 \pm 0.0$ & $100.0 \pm 0.0$ \\
\hline \multicolumn{3}{|l|}{ Catla } \\
\hline Initial total weight $\left(\mathrm{kg} \bullet\right.$ pond $\left.^{-1}\right)$ & $0.22 \pm 0.01$ & $0.21 \pm 0.00$ \\
\hline Initial mean weight $\left(\mathrm{g} \bullet \mathrm{fish}^{-1}\right)$ & $27.27 \pm 1.00$ & $25.87 \pm 0.26$ \\
\hline Final total weight $\left(\mathrm{kg}^{\circ}\right.$ pond $\left.^{-1}\right)$ & $0.91 \pm 0.04$ & $1.12 \pm 0.06$ \\
\hline Final mean weight $\left(\mathrm{g} \bullet \mathrm{fish}^{-1}\right)$ & $113.43 \pm \mathrm{a} 5.33$ & $153.40 \pm 14.03$ \\
\hline Daily weight gain $\left(\mathrm{g} \bullet \mathrm{fish}^{-1} \bullet \mathrm{day}^{-1}\right)$ & $0.96 \pm 0.07^{\mathrm{b}}$ & $1.42 \pm 0.15^{\mathrm{a}}$ \\
\hline Total weight gain $\left(\mathrm{kg}^{\circ}\right.$ pond $\left.{ }^{-1}\right)$ & $0.69 \pm 0.05^{\mathrm{b}}$ & $0.91 \pm 0.06^{\mathrm{a}}$ \\
\hline Survival (\%) & $100.0 \pm 0.0$ & $91.7 \pm 4.2$ \\
\hline \multicolumn{3}{|l|}{ Mrigal } \\
\hline Initial total weight $\left(\mathrm{kg} \bullet\right.$ pond $\left.^{-1}\right)$ & $0.20 \pm 0.00$ & $0.20 \pm 0.02$ \\
\hline Initial mean weight $\left(\mathrm{g} \bullet \mathrm{fish}^{-1}\right)$ & $25.03 \pm 0.28$ & $25.60 \pm 2.16$ \\
\hline Final total weight $\left(\mathrm{kg}^{\circ}\right.$ pond $\left.{ }^{-1}\right)$ & $1.03 \pm 0.05$ & $1.31 \pm 0.09$ \\
\hline Final mean weight $\left(\mathrm{g} \bullet \mathrm{fish}^{-1}\right)$ & $135.00 \pm 5.54$ & $163.80 \pm 11.49$ \\
\hline Daily weight gain $\left(\mathrm{g} \bullet \mathrm{fish}^{-1} \bullet \mathrm{day}^{-1}\right)$ & $1.22 \pm 0.06$ & $1.54 \pm 0.12$ \\
\hline Total weight gain $\left(\mathrm{kg}^{\circ}\right.$ pond $\left.{ }^{-1}\right)$ & $0.83 \pm 0.05$ & $1.11 \pm 0.09$ \\
\hline Survival $(\%)$ & $100.0 \pm 0.0$ & $100.0 \pm 0.0$ \\
\hline \multicolumn{3}{|l|}{ Common } \\
\hline Initial total weight $\left(\mathrm{kg} \bullet\right.$ pond $\left.^{-1}\right)$ & $0.21 \pm 0.01$ & $0.23 \pm 0.04$ \\
\hline Initial mean weight $\left(\mathrm{g} \bullet \mathrm{fish}^{-1}\right)$ & $26.63 \pm 1.54$ & $28.77 \pm 4.47$ \\
\hline Final total weight $\left(\mathrm{kg}^{\circ}\right.$ pond $\left.^{-1}\right)$ & $1.25 \pm 0.08$ & $1.16 \pm 0.13$ \\
\hline Final mean weight $\left(\mathrm{g} \bullet \mathrm{fish}^{-1}\right)$ & $172.43 \pm 8.72$ & $145.17 \pm 15.98$ \\
\hline Daily weight gain $\left(\mathrm{g} \bullet \mathrm{fish}^{-1} \bullet \mathrm{day}^{-1}\right)$ & $1.62 \pm 0.09$ & $1.29 \pm 0.22$ \\
\hline Total weight gain $\left(\mathrm{kg}^{\circ}\right.$ pond $\left.\mathrm{d}^{-1}\right)$ & $1.04 \pm 0.07$ & $0.93 \pm 0.16$ \\
\hline Survival (\%) & $95.8 \pm 4.2$ & $100.0 \pm 0.2$ \\
\hline \multicolumn{3}{|l|}{ Silver } \\
\hline Initial total weight $\left(\mathrm{kg} \cdot\right.$ pond $\left.^{-1}\right)$ & $0.13 \pm 0.00$ & $0.13 \pm 0.01$ \\
\hline Initial mean weight $\left(g \bullet f_{i s h}{ }^{-1}\right)$ & $31.70 \pm 0.91$ & $32.00 \pm 3.08$ \\
\hline Final total weight $\left(\mathrm{kg}^{\circ}\right.$ pond $\left.{ }^{-1}\right)$ & $1.42 \pm 0.10$ & $1.67 \pm 0.14$ \\
\hline Final mean weight $\left(\mathrm{g} \bullet \mathrm{fish}^{-1}\right)$ & $387.73 \pm 24.47$ & $417.37 \pm 34.93$ \\
\hline Daily weight gain $\left(\mathrm{g} \bullet \mathrm{fish}^{-1} \bullet \mathrm{day}^{-1}\right)$ & $3.96 \pm 0.28$ & $4.28 \pm 0.38$ \\
\hline Total weight gain $\left(\mathrm{kg}^{\circ}\right.$ pond $\left.^{-1}\right)$ & $1.29 \pm 0.10$ & $1.54 \pm 0.14$ \\
\hline Survival (\%) & $100.0 \pm 0.0$ & $100.0 \pm 0.0$ \\
\hline
\end{tabular}


S. Rai, Y.Yi, Md.A. Wahab, A.N. Bart and J.S. Diana / Our Nature (2010) 8: 92-105 Table 6-Contd....

\begin{tabular}{lll}
\hline Combined & & \\
Initial total weight $\left(\mathrm{kg} \bullet\right.$ pond $\left.^{-1}\right)$ & $1.04 \pm 0.02$ & $1.05 \pm 0.04$ \\
Final total weight $\left(\mathrm{kg} \bullet\right.$ pond $\left.^{-1}\right)$ & $6.28 \pm 0.32$ & $7.14 \pm 0.02$ \\
Total weight gain $\left(\mathrm{kg} \bullet\right.$ pond $\left.^{-1}\right)$ & $5.24 \pm 0.32$ & $6.09 \pm 0.06$ \\
FCR & $1.2 \pm 0.1$ & $1.1 \pm 0.0$ \\
\hline
\end{tabular}

Mean values with different superscript letters in the same row are significantly different $(\mathrm{P}<0.05)$.

Table 7. Gross margin analys is of different treatments based on $40 \mathrm{~m}^{2}$ pond in Bangladeshi currency Taka

\begin{tabular}{|c|c|c|c|c|c|c|}
\hline \multirow{2}{*}{ Item } & \multirow{2}{*}{ Unit } & \multirow{2}{*}{ Taka•unit $^{-1}$} & \multicolumn{2}{|c|}{ Rice straw } & \multicolumn{2}{|c|}{ Kanchi } \\
\hline & & & Quantity & Taka & Quantity & Taka \\
\hline \multicolumn{7}{|l|}{ Gross return } \\
\hline Rohu & $\mathrm{kg}$ & 60 & 1.73 & $104 \pm 7$ & 1.89 & $113 \pm 3$ \\
\hline Catla & $\mathrm{kg}$ & 60 & 0.91 & $54 \pm 3^{b}$ & 1.12 & $67 \pm 3^{a}$ \\
\hline Mrigal & $\mathrm{kg}$ & 60 & 1.03 & $62 \pm 3$ & 1.31 & $79 \pm 6$ \\
\hline Common & $\mathrm{kg}$ & 60 & 1.25 & $75 \pm 9$ & 1.16 & $70 \pm 13$ \\
\hline Silver & $\mathrm{kg}$ & 60 & 1.42 & $85 \pm 10$ & 1.67 & $100 \pm 15$ \\
\hline Total gross return & & & & $381 \pm 31^{b}$ & & $429 \pm 2^{\mathrm{a}}$ \\
\hline
\end{tabular}

\section{Variable cost}

Fingerlings

\begin{tabular}{|c|c|c|c|c|c|c|}
\hline Rohu & Pcs & 3.5 & 12 & 42 & 12 & 42 \\
\hline Catla & Pcs & 3.5 & 8 & 28 & 8 & 28 \\
\hline Mrigal & Pcs & 3.5 & 8 & 28 & 8 & 28 \\
\hline Common & Pcs & 3.5 & 8 & 28 & 8 & 28 \\
\hline Silver & Pcs & 3.5 & 4 & 14 & 4 & 14 \\
\hline \multicolumn{7}{|l|}{ Feed } \\
\hline Rice bran & $\mathrm{kg}$ & 10 & 4.1 & 41 & 4.8 & 48 \\
\hline Mustard oil cake & $\mathrm{kg}$ & 15 & 3.0 & 45 & 3.1 & 47 \\
\hline \multicolumn{7}{|l|}{ Fertilizer } \\
\hline Urea & $\mathrm{kg}$ & 8 & 0.868 & 7 & 0.868 & 7 \\
\hline TSP & $\mathrm{kg}$ & 15 & 0.448 & 7 & 0.448 & 7 \\
\hline Cowdung & $\mathrm{kg}$ & 0.4 & 35 & 14 & 35 & 14 \\
\hline Lime & $\mathrm{kg}$ & 12 & 1 & 12 & 1 & 12 \\
\hline Kanchi & Pcs & 1 & - & - & $\begin{array}{l}390 \text { for } 4 \\
\text { crops }\end{array}$ & 98 \\
\hline Bamboo & Pcs & 130 & $\begin{array}{l}2 \text { for } 9 \\
\text { crops }\end{array}$ & 29 & - & - \\
\hline Wire & & & & 20 & & \\
\hline $\begin{array}{l}\text { Interest on working } \\
\text { capital }\end{array}$ & & $10 \%$ & & 8 & & 9 \\
\hline Total variable cost & & & & $322 \pm 0$ & & $381 \pm 0$ \\
\hline Gross margin & & & & $59 \pm 18$ & & $48 \pm 1$ \\
\hline
\end{tabular}

Mean values with different superscript letters in the same row are significantly different $(\mathrm{P}<0.05)$. 


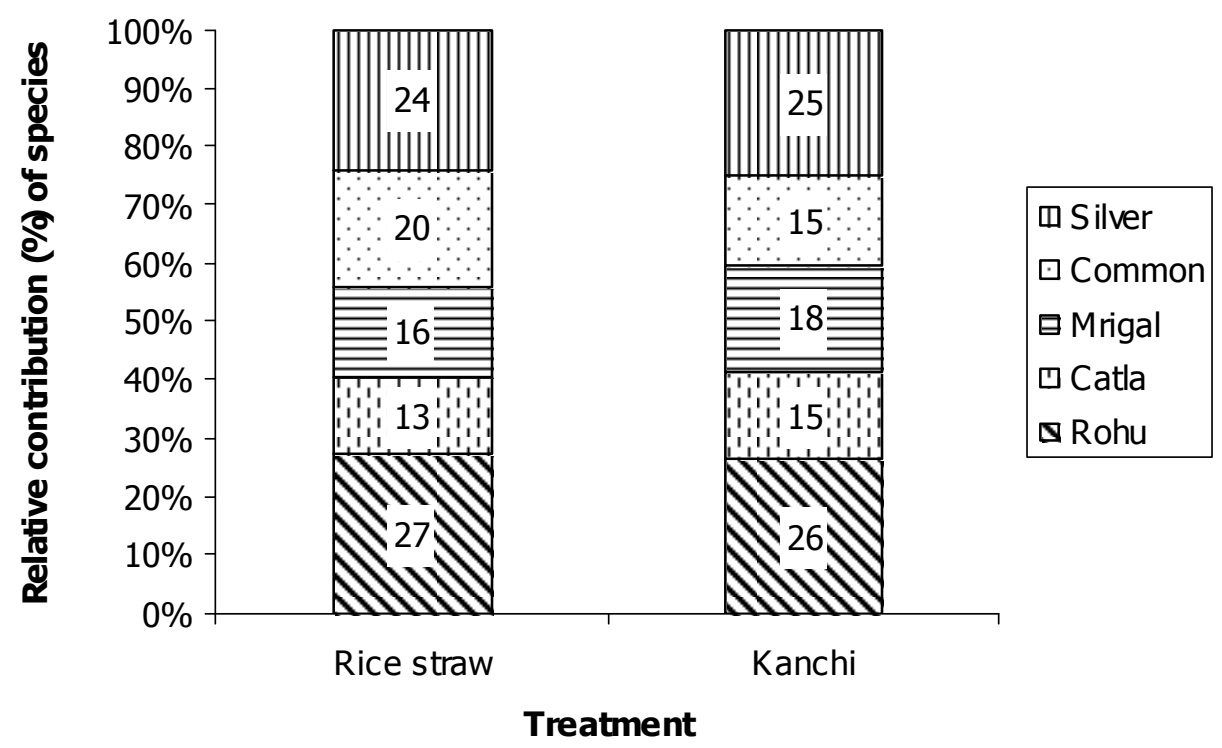

Figure 1. Relative contribution of different species on combined total weight gain in the rice straw and kanchi treatments.

the present experiment, combined total weight gains were higher than that reported by Azim et al. (2001b) and Van Dam et al. (2002) for the potential yield from periphyton-based aquaculture system. Among the stocked carps, silver carp contributed more than $24 \%$ of the total weight gain in both treatments, though it represented only $10 \%$ of the population in the present experiment. Silver carp grew better because it is an efficient filter feeder (Milstein et al., 1985). FCR did not differ between the rice straw and kanchi treatments because feed was provided based on fish biomass and fish production was not significantly different between the treatments. FCR in the present experiment was lower than that reported by Sahu et al. (2007).
Gross margin analysis showed that both treatments were profitable. Gross margin was higher in the rice straw treatment than that in the kanchi treatment due probably to low cost of rice straw. Since the rice straw in the ponds with supplemental feed gave fish production as high as in the kanchi ponds with supplemental feed, the rice straw treatment seemed better for the resource-poor farmers. As rice straw decomposes gradually, further research is needed on using rice straw as substrate for long term fish culture.

\section{Acknowledgements}

This research is a component of the Aquaculture Collaborative Research Support Program (A/CRSP), supported by the US Agency for International 
Development Grant No. LAG-G-00-9690015-00, and by contributions from the University of Michigan, the Asian Institute of Technology, and Bangladesh Agricultural University. The authors wish to thank Prof. M.B. Rahman for guiding Microbiology work, Associate Prof. Dr. Z.F. Ahmed, Mr. A. Masud, Mr. Shaheen, Mr. Md. Asaduzzaman and students of Water Quality and Pond Dynamics Laboratory, Field Lab staffs of Fisheries Faculty and staffs of Central Laboratory, Bangladesh Agricultural University, Mymensingh for their help during this research, and Dr. T.B. Gurung and Research Scientists A. Rayamajhi and N. Pradhan of Fisheries Research Division, Nepal Agriculture Research Council for providing the laboratory facility and help to carry out periphyton analysis. The A/CRSP accession number is 1340. The opinions expressed herein are those of the authors and do not reflect the views of the US Agency for International Development.

\section{References}

APHA 1980. Standard methods for the examination of water and wastewater. $15^{\text {th }}$ edn. American Public Health Association, Washington D.C.

Azim, M.E., M.A. Wahab, A.A. Van Dam, M.C.M. Beveridge, M.C.J. Verdegem and A.A. Van Dam 2001a. The potential of periphyton based culture of two Indian major carps, rohu, Labeo rohita (Hamilton) and gonia, Labeo gonius (Linnaeus). Aquacul. Res. 32: 209216.

Azim, M.E., M.A. Wahab, A.A. van Dam, M.C.M. Beveridge, A. Milstein and M.C.J. Verdegem 2001b. Optimization of fertilization rate for maximizing periphyton production on artificial substrates and implications for periphyton-based aquaculture. Aquacul. Res. 32: 749-760.

Azim, M.E., M.A. Wahab, P.K. Biswas, T. Asaeda, T. Fujino and M.C.J. Verdegem 2004. The effect of periphyton substrate density on production in freshwater polyculture ponds. Aquaculture 232: 441-453.

Azim, M.E., M.C.J. Verdegem, H. Khatun, M.A. Wahab, A.A. van Dam and M.C.M. Beveridge 2002b. A comparison of fertilization, feeding and three periphyton substrates for increasing fish production in freshwater pond aquaculture in Bangladesh. Aquaculture 212: 227-243

Azim, M.E., M.C.J. Verdegem, M.M. Rahman, M.A. Wahab, A.A. van Dam and M.C.M. Beveridge 2002a. Evaluation of polyculture with Indian major carps in periphyton-based pond. Aquaculture 213: 131-149.

Bellinger, E.G. 1992. A key to common algae. The Institute of Water and Environmental Management, London.

Chakrabarty, N.M. 1998. Biology, culture and production of Indian major carp- A review. Narendra Publishing House, Delhi, India.

Dharmaraj, M., J.K. Manissery and P. Keshavnath 2002. Effects of a biodegradable substrate, sugarcane bagasse and supplementary feed on growth and production of fringe-lipped peninsular carp, Labeo fimbriatus (Bloch). Acta Ichthyologica et Piscatoria 32: 137-144.

Habib, A.B., M. Ahmed, M.A. Haque and A.K.M.A. Islam 1984. Status of macrobenthos in two ponds in Bangladesh Agricultural University Campus, Mymensingh. Bangla. J. Agr. Sci. 11: 39-46.

Hem, S. and J.L.B. Avit 1994. First results on 'acadjaenclos' as an extensive aquaculture system, Cote d'Ivoire (West Africa). Bull. Maricul. Sci. 55: 1040-1051.

Keshavnath, P., B. Ganghadhar, T.J. Ramesh, A.A. Van Dam, M.C.M. Beveredge and M.C.J. Verdegem 2002. The effect of periphyton and feeding on the production of the indigenous carps Tor khudree and Labeo fimbratus. Aquaculture 213: 207-218.

Keshavnath, P., B. Ganghadhar, T.J. Ramesh, A.A.Van Dam, M.C.M. Beveredge and M.C.J. Verdegem 2001. On farm evaluation of Indian major carp production with sugarcane bagasse as substrate for periphyton. Asi. Fish. Sci. 14: 367-376. 
Langis, R., D. Proulx, J. de la Noue and P. Couture 1988. Effects of a bacterial biofilm on I Daphnia culture. Aquacul. Engineer. 7: 2138.

Milstein, A. 1992. Ecological aspects of fish species interactions in polyculture ponds. Hydrobiol. 231: $17-186$.

Milstein, A., B. Hepher and B. Teltsch 1985. Interactions between fish species and the ecological conditions in mono and polyculture pond system. Aquacul. Fish. Manag. 16: 305317.

Moriarty, D.J.W. 1986. Bacterial productivity in ponds for culture of penaeid prawns. Microbiol. Ecol. 12: 259-270.

Moriarty, D.J.W. 1997. The role of microorganisms in aquaculture ponds. Aquaculture 151: 333349.

Mridula, R.M., J.K. Manissary, P. Keshavnath, K.M. Shankar, M.C. Nandesha and K.M. Rajesh 2003. Water quality, biofilm production and growth of fringe-lipped carp (Labeo fimbriatus) in tanks provided with two solid substrates. Aquaculture 87: 263-267.

Mridula, R.M., J.K. Manissary, P. Keshavnath, K.M. Shankar, M.C. Nandesha and K.M. Rajesh 2005. Effects of paddy straw and sugarcane bagasse on water quality, bacterial biofilm production and growth and survival of rohu, Labeo rohita (Hamilton). Aquacul. Res. 36: 635-642.

Needham, J.G. and P.R. Needham 1962. A guide to the study of fresh-water biology. Holden-Day, Inc., San Francisco.

Neori, A. and M.D. Krom 1991. Nitrogen and phosphorous budgets in an intensive marine fishpond: the importance of microplankton. In Nutritional Strategies and Aquaculture Waste (Eds. C.V. Cowey and C.Y. Cho). pp. 223230.

Rahman, M.M., M.C.J. Verdegem, L.A.J. Nagelkerke, M.A. Wahab, A. Milstein and J.A.J. Verreth 2006. Growth production and food preference of rohu Labeo rohita $(\mathrm{H})$ in monoculture and in polyculture with common carp Cyprinus carpio (L.) under fed and non-fed ponds. Aquaculture 257: 359-372.
Ramesh, M.R., K.M. Shankar, C.V. Mohan and T.J. Varghese 1999. Comparison of three plant substrates for enhancing carp growth through bacterial biofilm. Aquacul. Engineer. 19: 119-131.

Raveh, A. and Y. Avnimelech 1979. Total nitrogen analysis in water, soil and plant material with persulfate digestion. Wat. Res. 13: 911-912

Sahu, P.K., J.K. Jena, P.C. Das, S. Mondal and R. Das 2007. Production performance of Labeo calbasu (Hamilton) in polyculture with three Indian major carps Catla catla (Hamilton), Labeo rohita (Hamilton) and Cirrhinus mrigala (Hamilton) with provision of fertilizers, feed and periphytic substrate as varied inputs. Aquaculture 262: 333-339.

Schroeder, G.L. 1978. Autotrophic and heterotrophic production of micro-organisms in intenselymanured fish ponds, and related fish yields. Aquaculture 14: 303-325.

Siddiqui, A.Q. and A.H. Al-Harbi 1999. Nutrients budgets in tanks with different stocking densities of hybrid tilapia. Aquaculture 170: 245-252.

Tang, Y.A. 1970. Evaluation of balance between fishes and available fish foods in multispecies fish culture ponds in Taiwan. Trans Amer. Fish Soc. 4: 708-718.

Van Dam, A.A., M.C.M. Beveridge, M.E. Azim and M.C.J. Vedegem 2002. The potential of fish production based on periphyton. Rev. Fish Biol. Fisheries 12: 1-31.

Wahab, M.A., Z.F. Ahmed, M.A. Islam, M.S. Haq and S.M. Ramatullah 1999. Effects of introduction common carp, Cyprinus carpio (L.) on pond ecology and growth of fish in polyculture. Aquacul. Res. 26: 619-628.

Ward, H.B. and G.C. Whipple 1959. Freshwater Biology. John Willey and sons Inc., New york.

Wetzel, R.G. 1983. Attached algal-substrata interactions; fact or myth, and when and how? In Periphyton of freshwater ecosystems (Ed. R.G. Wetzel), Dr. W. Junk publishers, The Hague. pp. 207-215. 